\title{
Review Article \\ Treatment of Dermatological Conditions Associated with HIV/AIDS: The Scarcity of Guidance on a Global Scale
}

\author{
Suchismita Paul, ${ }^{1}$ Rachel Evans, ${ }^{2}$ Toby Maurer, ${ }^{3}$ Lulu M. Muhe, ${ }^{4}$ and Esther E. Freeman ${ }^{5}$ \\ ${ }^{1}$ Harvard Medical School, Boston, MA, USA \\ ${ }^{2}$ HIV Department, World Health Organization, Geneva, Switzerland \\ ${ }^{3}$ Department of Dermatology, University of California San Francisco School of Medicine, San Francisco, CA, USA \\ ${ }^{4}$ Department of Maternal, Child and Adolescent Health, World Health Organization, Geneva, Switzerland \\ ${ }^{5}$ Department of Dermatology, Massachusetts General Hospital, Harvard Medical School, Bartlett Hall 6R, 55 Fruit Street, \\ Boston, MA 02114, USA \\ Correspondence should be addressed to Esther E. Freeman; esther.freeman@gmail.com
}

Received 24 September 2015; Accepted 2 March 2016

Academic Editor: Andrea Mangano

Copyright ( 2016 Suchismita Paul et al. This is an open access article distributed under the Creative Commons Attribution License, which permits unrestricted use, distribution, and reproduction in any medium, provided the original work is properly cited.

\begin{abstract}
Background. Skin diseases associated with Human Immunodeficiency Virus (HIV) infection are associated with significant morbidity and mortality. In resource-limited settings, nondermatologists and lay health care providers on the front line of HIV care provide much of the treatment for these conditions. Objective. To evaluate guidelines for treatment of HIV-related skin conditions and assess their accessibility, comprehensiveness, and quality of evidence employed. Methods. A review was undertaken of all national and society guidelines which included treatment information on the ten highest burden HIV-related skin conditions. The search strategy included gray and peer-reviewed literature. Results. Of 430 potential guidelines, 86 met inclusion criteria, and only 2 were written specifically to address HIV-related skin diseases as a whole. Treatment information for HIV-related skin conditions was embedded within guidelines written for other purposes, primarily HIV/AIDs treatment guidelines (49\%). Development of guidelines relied either partially or completely on expert opinion (62\%). Only $16 \%$ of guidelines used gradation of evidence quality and these were primarily from high-income countries $(p=0.001)$. Limitations. Due to the nature of gray literature, not all guidelines may have been identified. Conclusion. This review highlights the need for evidence-based summary guidelines that address treatment for HIV-related skin conditions in an accessible format.
\end{abstract}

\section{Introduction}

In 2013, the number of people living with Human Immunodeficiency Virus (HIV) was estimated to be 35 million (31.8 million adults and 3.2 million children $<15$ years) globally [1]. There were 2.1 million new HIV infections (1.9 million adults and 240,000 children) and 1.5 million deaths due to HIV-related causes (1.3 million adults and 190,000 children) [1]. The major causes of mortality are Acquired Immunodeficiency Syndrome (AIDS) related mortality or from opportunistic infections such as tuberculosis (TB) and cryptococcal infections. The initiation of combination antiretroviral therapy (cART) has led to a profound impact on mortality, averting 7.6 million deaths globally since 1995 [1].
Globally, skin conditions are the fourth leading cause of nonfatal disease burden in terms of years lost due to disability, ahead of conditions such as diabetes and COPD [2]. HIVrelated skin conditions are important in terms of burden, impact on quality of life, and associated mortality. Prior to availability of effective cART, it was estimated that up to 90\% of HIV-infected individuals have associated skin and mucosal conditions during the course of their illness [3]. Skin manifestations may be the first sign of HIV infection and therefore present an opportunity for HIV testing and earlier diagnosis [3]. Some mucocutaneous conditions in particular are a proxy indicator for more advanced immunodeficiency and the need for prompt initiation of cART $[4,5]$. Certain skin conditions can also lead to severe morbidity such as pain on swallowing from oropharyngeal candidiasis and recurrent 
infections that are difficult to treat, such as scabies [6, 7]. Other conditions such as zoster and extensive tinea that are difficult to conceal may cause stigma and cause psychosocial stress and depression [8].

Particular challenges in the effective management of HIV-related skin conditions include the fact that they are difficult to treat and may recur more frequently compared to immunocompetent individuals in the absence of immune reconstitution with cART $[9,10]$. Early recognition of HIVrelated skin conditions presents the opportunity for earlier HIV diagnosis and cART initiation and may therefore improve overall survival [11]. In addition, in resource-limited settings, the focus is on more life-threatening opportunistic infections and skin conditions may be overlooked [12]. Finally, in many countries there is a lack of specialized dermatologists and the front line provision of HIV care is by primary care level nonspecialists that include medical officers, nurses, and midwives with minimal training in treatment of skin conditions $[7,13]$.

There is a need for clear practical but evidence-based guidance on the management of skin conditions in HIVinfected individuals. In this paper we review availability of national and professional society guidelines on HIV-related skin conditions and assess their accessibility, comprehensiveness, and the quality of evidence employed.

\section{Methods}

2.1. Search Strategy. We formulated a structured and comprehensive search strategy, using both peer-reviewed and gray literature as described in more detail below, to identify treatment guidelines for HIV-related skin conditions. Gray literature encompasses publications from governments, nongovernmental organizations, and societies that usually do not fulfil strict bibliographical requirements that are apparent in peer-reviewed literature [14]. The inclusion of gray literature sources was key in this process, since guidelines are not consistently or routinely included in peer-reviewed literature or in databases such as PubMed. Treatment guidelines were defined as a set of recommendations used to treat skin conditions in HIV-infected patients. Guidelines for children, adolescents, and adults were included as well as guidelines written in languages other than English.

2.2. Gray Literature Search. The gray literature search was performed in June 2014 and was divided into two categories: national guidelines and society guidelines. National guidelines were defined as guidelines developed by federal governments. From the UNAIDS database, 30 countries with highest HIV prevalence and 30 countries with the highest estimated number of people living with HIV were included [15]. Both lists were merged and duplicates were eliminated to yield a total of 43 countries. An additional 7 countries active in HIV/AIDS policy were selected on a discretionary basis to bring the list to a total of 50 countries (see the Appendix). The following specific databases along with web searches (Google, Google scholar) were used to obtain countryspecific guidelines: World Health Organization (WHO), database of national HIV and TB guidelines, 2005-2011 [16];
USAID, AIDSTAR-One, National Treatment Guidelines [17]; the Interagency Task Team on the Prevention and Treatment of HIV Infection in Pregnant Women, Mothers and Children [18]; AIDSspace, Document Library [19]; and the Ministry of Health website for each country.

Society guidelines were defined as guidelines developed by nonprofit, nongovernmental organizations such as the Infectious Disease Society of America (IDSA) or international agencies such as the WHO (see the Appendix). Guidelines were also included from societies in dermatology, infectious diseases, and HIV/AIDS. These were obtained from the organizations' webpages.

2.3. Peer-Reviewed Literature Search. The peer-reviewed literature search was carried out on PubMed using the Cochrane HIV/AIDS Group's existing validated strategies to identify articles relevant to HIV infection and AIDS along with $\mathrm{MeSH}$ terms and relevant keywords to identify treatment guidelines for associated skin conditions [20].

2.4. Inclusion Criteria. For both gray and peer-reviewed literature, duplicate guidelines and older versions of the same guideline were excluded. Guidelines were included if they mentioned the treatment of one or more of ten selected HIVrelated skin conditions: Kaposi's sarcoma, scabies, seborrheic dermatitis, molluscum contagiosum, eosinophilic folliculitis, papular pruritic eruption, varicella/herpes zoster, tinea, oropharyngeal candidiasis, and drug reactions (StevensJohnson syndrome or toxic epidermal necrolysis). Our search was restricted to ten skin conditions to allow us to perform an extensive gray literature search. These ten conditions are representative of other HIV-related skin conditions in terms of high disease burden, available evidence, effective interventions, and applicability in resource-limited settings.

2.5. Data Collection and Analysis of Guidelines. After screening and selection, we analyzed eligible guidelines with regard to the publication date, the frequency of specific skin conditions represented, the category of source document where the treatment for HIV-related skin conditions was mentioned, and the methodology that was used to develop guidelines. The type of guideline under which treatment guidelines for HIV-related skin conditions are found is an important factor to assess how quickly and easily a busy health care professional can access such treatment information. To address this issue of accessibility, we defined different types of guidelines as follows: HIV/AIDS treatment guidelines (guidelines that address treatment of HIV/AIDS using antiretroviral therapies), disease-specific treatment guidelines (treatment for one of the ten skin conditions associated with HIV such as scabies or tinea), STD/STI treatment guidelines (treatment of sexually transmitted infections (STDs)), skin disease treatment guidelines (treatment for skin diseases in general), opportunistic infections treatment guidelines (treatment for HIV-associated opportunistic infections), and standard clinical treatment guidelines (treatment for general medical conditions such as cardiovascular disorders and dermatological disorders). 


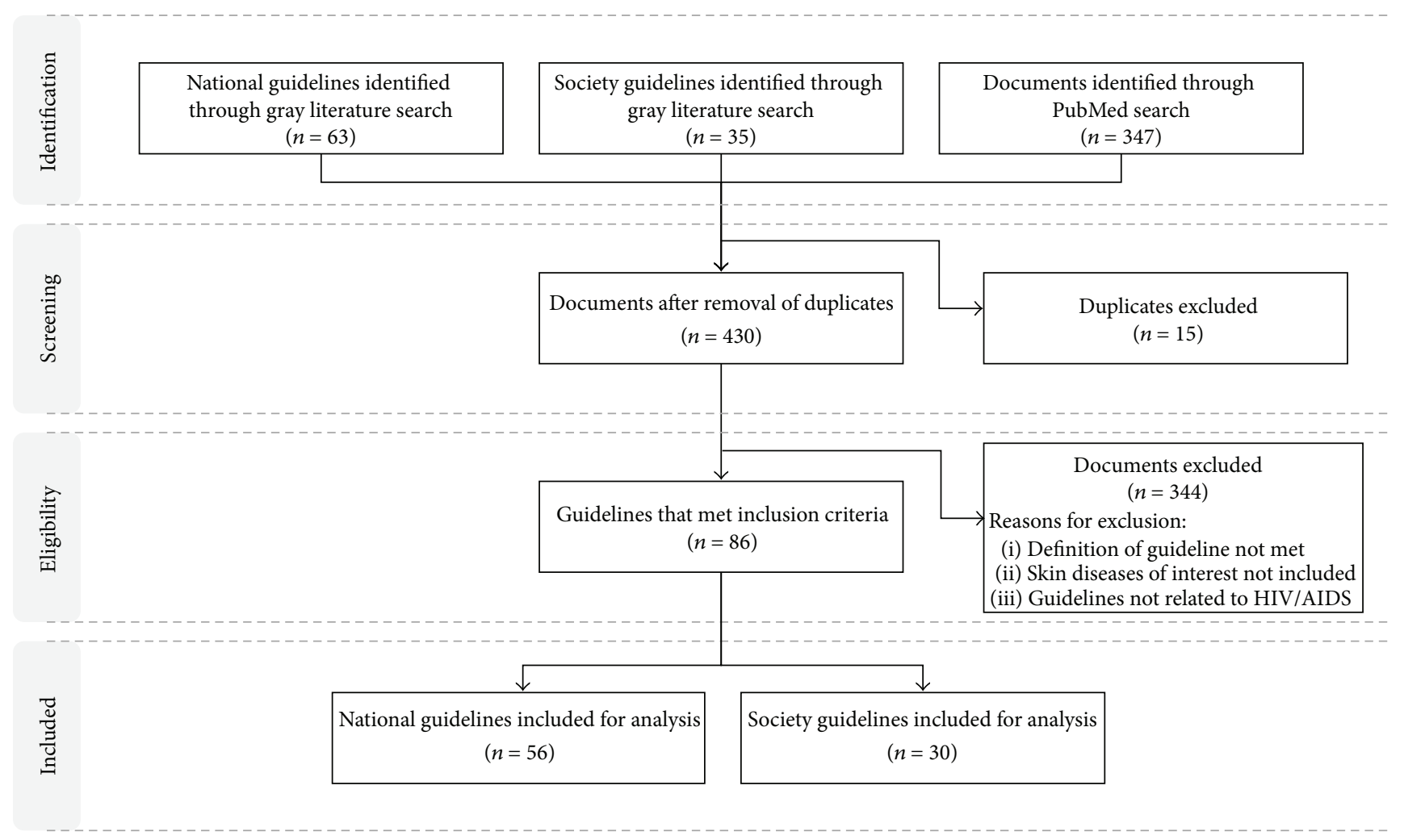

FIGURE 1: Prisma diagram showing selection process of treatment guidelines for HIV-related skin conditions.

We also assessed the methodology that was used to develop the treatment guidelines. We searched each document to determine which of the following methods were employed: expert opinion (based on experts' experience), scientific literature (based on results from clinical studies), graded evidence and strength of recommendations (based on rating systems such as Grading of Recommendations, Assessment, Development and Evaluation (GRADE) [21]), and adaptations from other guidelines (based on WHO and/or other guidelines).

2.6. Statistical Analysis. The countries were categorized by gross national income as defined by the World Bank: high income, middle income (upper middle and lower middle), and low income [22]. We tested the hypothesis that highincome countries would employ higher levels of evidence quality in their guideline development (Fisher's exact test).

\section{Results}

As of June 2014, the gray and peer-reviewed literature search yielded a total of 430 potential guidelines once duplicates were removed, of which 86 guidelines (56 national and 30 society guidelines) met our selection criteria related to treatment guidelines for HIV-related skin conditions (Figure 1). The society guidelines were obtained from organizations like the WHO, American Academy of Dermatology (AAD), British HIV Association, IDSA, and others (see the Appendix). Of the fifty countries assessed for national guidelines (see the Appendix), fifteen did not have national guidelines for the treatment of HIV-related skin conditions although Australia and the United Kingdom had guidelines from societies. Included guidelines were in English, Ukrainian, Indonesian, Spanish, Portuguese, French, and Chinese.

Guidelines identified ranged from the years 1997 to 2014, with almost half (45\%) more than five years old. Not all HIV-related skin conditions were included in each guideline. Among 86 total guidelines, oropharyngeal candidiasis, varicella/zoster, and Kaposi's sarcoma were most frequently addressed (62\%, 60\%, and 50\%, resp.), whereas eosinophilic folliculitis, tinea, and papular pruritic eruption were the least represented (9\%, $21 \%$, and $26 \%$, resp.).

3.1. Accessibility of Treatment Guidelines for HIV-Related Skin Conditions. Accessibility of the guidelines, defined as how easily treatment for HIV-related skin conditions are found, was poor. When searches of the gray literature were limited to guidelines that were specifically for HIV-related skin conditions, only two guidelines were identified, from AAD (1997) and the New York State Department of Health AIDS Institute $(2004)[23,24]$. In the remaining 84 guidelines, treatment for different HIV-related skin conditions was mentioned within the context of HIV treatment in general ( $n=41,49 \%)$, STD guidelines $(n=2,2 \%)$, skin disease treatment guidelines $(n=2,2 \%)$, standard clinical treatment guidelines $(n=14,17 \%)$, opportunistic infections treatment guidelines $(n=12,14 \%)$, and disease-specific treatment guidelines ( $n=13,15 \%)$ (Table 1). Among national guidelines 


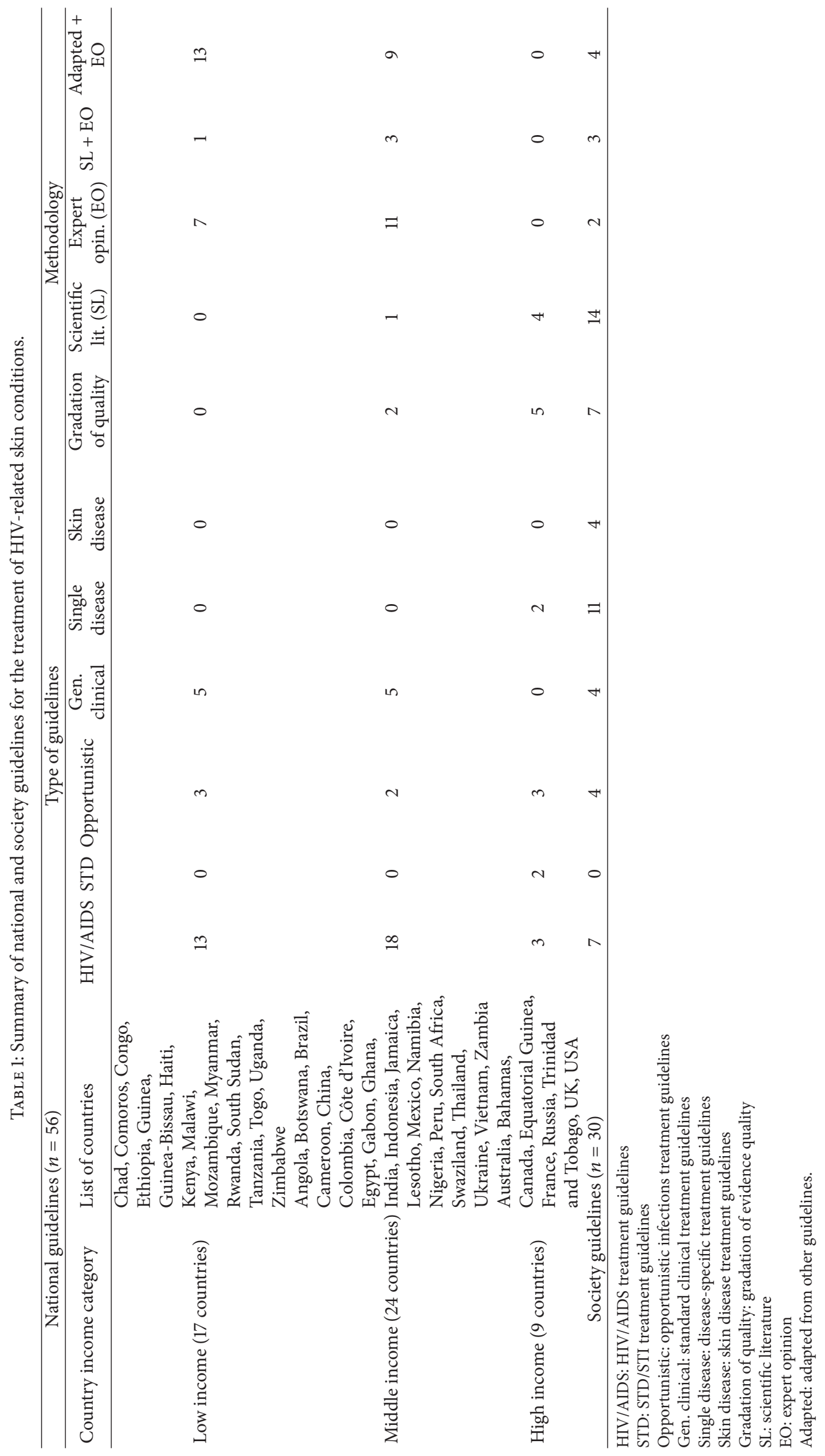




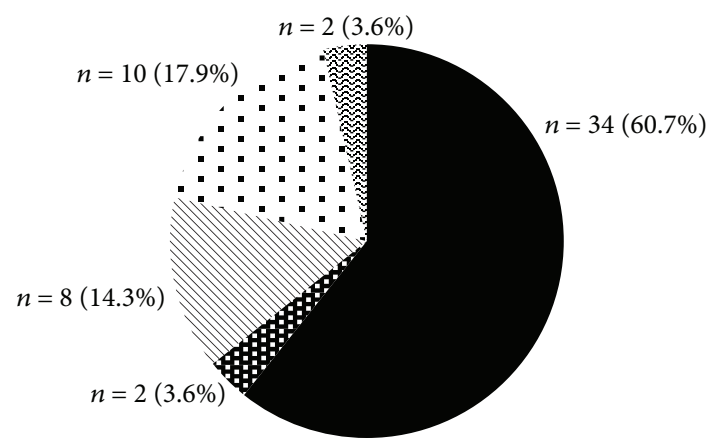

- HIV/AIDS treatment guidelines

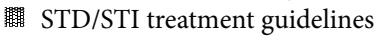
Opportunistic infections treatment guidelines : Standard clinical treatment guidelines 澡 Disease-specific treatment guidelines $\approx$ Skin disease treatment guidelines

(a) National guidelines

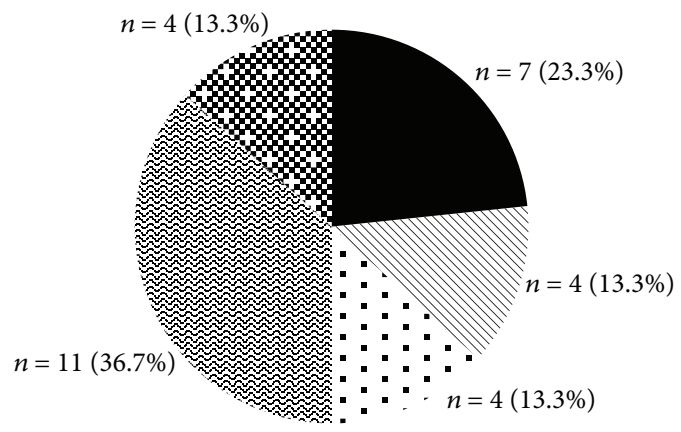

- HIV/AIDS treatment guidelines

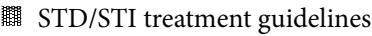
Opportunistic infections treatment guidelines Standard clinical treatment guidelines

注 Disease-specific treatment guidelines

Skin disease treatment guidelines

(b) Society guidelines

FIGURE 2: Categories of source documents where treatment of HIV-related skin conditions was mentioned.

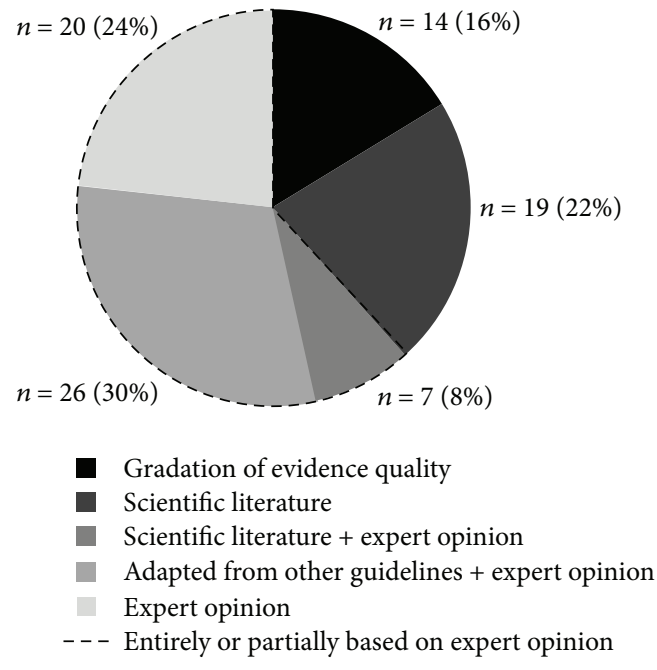

(a)

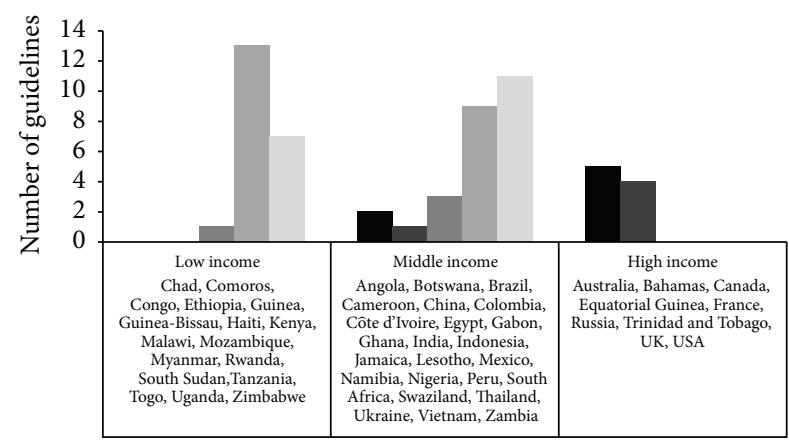

Gradation of evidence quality

Scientific literature

Scientific literature + expert opinion

- Adapted from other guidelines + expert opinion

Expert opinion

Figure 3: (a) Methodologies used to develop guidelines for HIV-related skin conditions $(n=86)$. (b) Association between gross national income of countries and the methodology used to develop guidelines for HIV-related skin conditions.

produced by specific countries' governments, the treatment guidelines for HIV-related skin conditions were found within five different types of guidelines, with the majority from HIV/AIDS treatment guidelines (61\%) and none from skin disease treatment guidelines (Figure 2(a)). In contrast, among guidelines produced by societies, this information was most frequently contained within the disease-specific treatment guidelines (37\%) (Figure 2(b)).

With respect to the ease of finding treatment guidelines for HIV-related skin conditions only 19 out of the 56 national guidelines had a dedicated dermatology section. For the remaining 37 national guidelines, treatment information for different HIV-related skin conditions was dispersed throughout the guideline.
3.2. Methodology Used to Develop Guidelines. There was a wide variability in the methods used to develop the treatment guidelines for HIV-related skin conditions, with most relying on expert opinion either partially or completely $(n=53,62 \%)$ rather than evidence-based scientific literature (Figure 3(a), Table 1). Guidelines frequently combined multiple methodologies such as adaptation from other guidelines along with expert opinion $(30 \%)$ or scientific literature combined with expert opinion (8\%). Only 14 guidelines (16\%) employed the highest quality of guideline development process, which involves a rating system to grade the quality of evidence. The rating systems varied and included GRADE, Oxford Centre for Evidence-Based Medicine (CEBM), US Preventative Services Task Force (USPSTF), IDSA/US Public Health Service (IDSA/USPHS), or adaptations thereof. 
Gross national income was correlated with the guideline methodology employed by national governments (Figure 3(b)). Low-income countries primarily adapted their guidelines from other sources (13 of 21) or relied on expert opinion (7 of 21). Middle-income countries relied on expert opinion (11 of 26) and adaptation from other guidelines (9 of 26), while a minor subset employed scientific literature (1 of 26 ) and gradation of evidence quality (2 of 26). High-income countries had either reviewed the scientific literature or used the gold standard of guideline development, gradation of evidence quality. High-income countries were significantly more likely to have employed an assessment of evidence quality in their guidelines (5 of 9 guidelines) as compared to lower- and middle-income countries ( 2 of 47 guidelines; $p=0.001)$.

\section{Discussion}

Our study suggests that there is a paucity of comprehensive evidence-based guidelines that is specific for treatment of HIV-related skin conditions. Currently, either HIV-related skin condition treatment information is difficult to find within the different types of guidelines or the methodology used to prepare the guidelines is not based on clinical evidence. Additionally, many guidelines are outdated and information across the prominent HIV-related skin conditions is fragmented across several guidelines. Together, these gaps highlight the need for an evidence-based, easily accessible summary guideline document for HIV-related skin conditions.

For a lay searcher or busy health professional, accessibility of current guidelines is poor. Firstly, to find treatment regimens for HIV-related skin conditions, entire guidelines written for other purposes such as HIV/AIDS treatment or opportunistic infections must be hand-searched. This process is time-consuming and represents a barrier to access by busy health care professionals. Furthermore, information for the major HIV-related skin conditions is scattered across several guidelines, requiring reference to multiple guidelines when trying to develop a treatment plan.

The guidelines identified rarely used evidence-based medicine. Methodology for guideline development varied widely, including a large subset based completely or partially on expert opinion (62\%). Treatment decisions based on unvalidated information may be more harmful than helpful and may lead to increased morbidity and even mortality $[21,25]$. Generally, treatment of skin conditions is often not evidence-based due to the lack of high quality studies and reliance on expert opinion is warranted under these circumstances. Further studies are needed to see whether recommendations differ between expert opinions and evidencebased medicine for treatment of skin conditions. In the smaller portion of guidelines that did employ gradation of evidence quality (16\%), there were several grading systems, including GRADE, IDSA, CEBM, and USPSTF. Highincome countries were much more likely to employ evidencebased medicine, in contrast to lower-income countries with higher HIV prevalence rates and arguably more in need of quality guidelines. In low-income countries, guidelines were primarily adapted from the WHO. This reliance on global guidance further highlights the need for high quality international guidelines.

Finally, almost half of the guidelines analyzed are more than five years old, which limits healthcare professionals to using dated information to address HIV-related skin conditions. Guidelines should be reassessed for validity and updated every 3 years for it to be useful to clinicians [26].

Our study has several limitations. With gray literature research, it is possible to miss guidelines due to the nature of gray literature records that might not be accessible through conventional searches. Our search was performed in June 2014 and guidelines that became available online since then could have been missed. Additionally, only documents which specified "HIV" or "AIDS" were included, whereas general immune deficiency or impairment was not considered. Guidelines written in local languages might also have been overlooked because they were not searchable in English. Finally, we restricted the review to the 10 highest burden $\mathrm{HIV}$-related skin conditions and the selected 50 countries. We make the assumption that these parameters are representative of HIV-related skin condition treatment guidelines globally.

This review highlights the need for an effective guideline document for busy healthcare professionals to treat HIVrelated skin conditions and identifies a gap in guidelines development within the field of dermatology. A comprehensive treatment guideline for HIV-related skin conditions should be a compilation of the most up-to-date treatment recommendations that are strictly vetted through a rating system for the evidence quality and strength of recommendations (such as GRADE) [21]. Recurrent updating would arm healthcare professionals with the latest treatment information. In response to these previously unmet needs, as identified in this work, the WHO has developed new set of guidelines for the treatment of the ten HIV-related skin conditions that were discussed in this study [27]. These guidelines were developed using the Cochrane systematic review and the GRADE rating system. Additionally, these guidelines address the local needs and constraints within resourcelimited settings by considering factors such as availability of medications and costs of drugs. With the adoption of such guidelines, which can be accessed and adapted to different health systems, we can hope to see a decrease in morbidity and mortality related to HIV-related skin conditions.

\section{Appendix}

See Tables 2 and 3 .

\section{Abbreviations}

AAD: American Academy of Dermatology

AIDS: Acquired Immunodeficiency Syndrome

cART: Combination antiretroviral therapy

CEBM: Centre for Evidence-Based Medicine

COPD: Chronic obstructive pulmonary disease

GRADE: Grading of Recommendations, Assessment, Development and Evaluation

HIV: Human Immunodeficiency Virus 


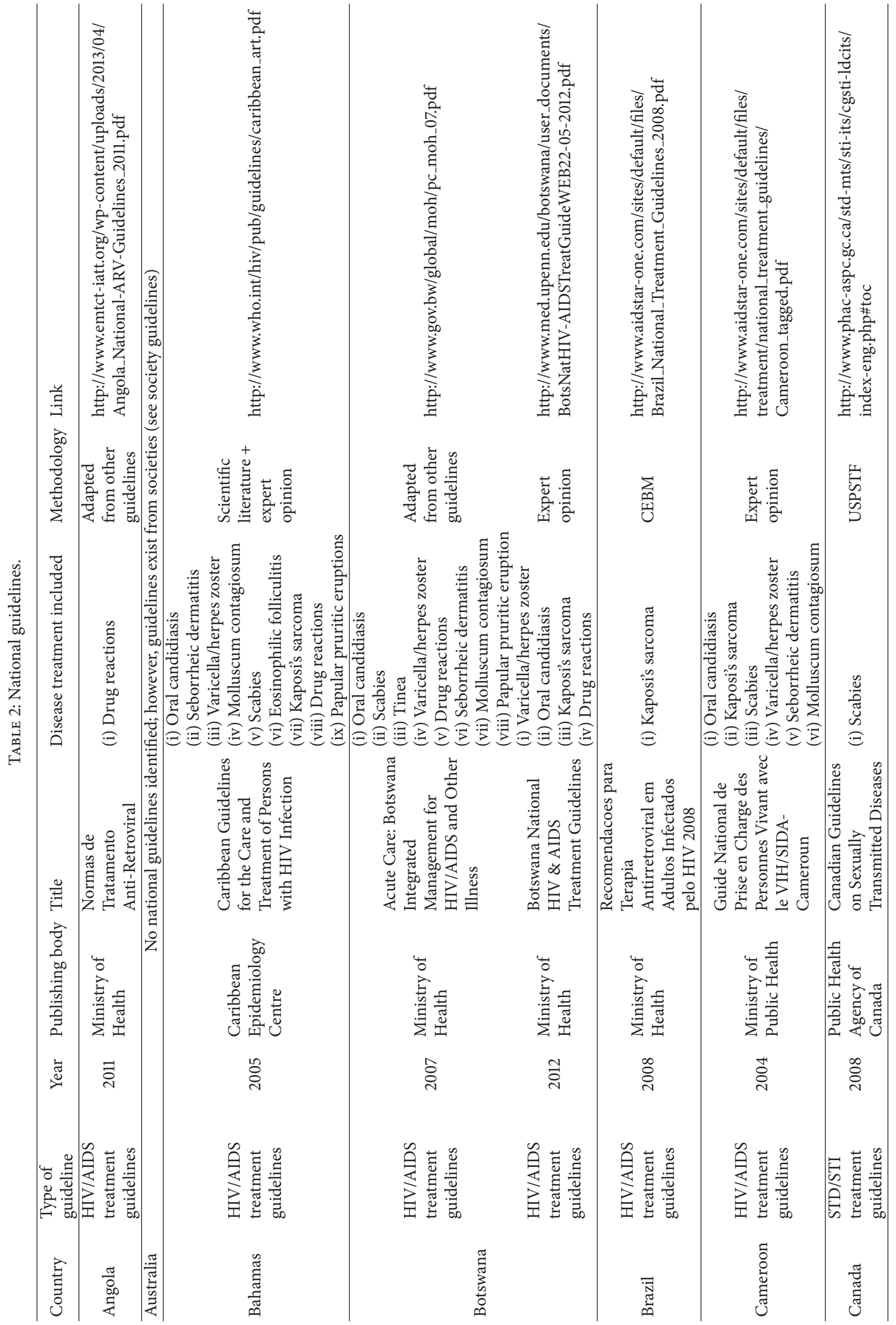




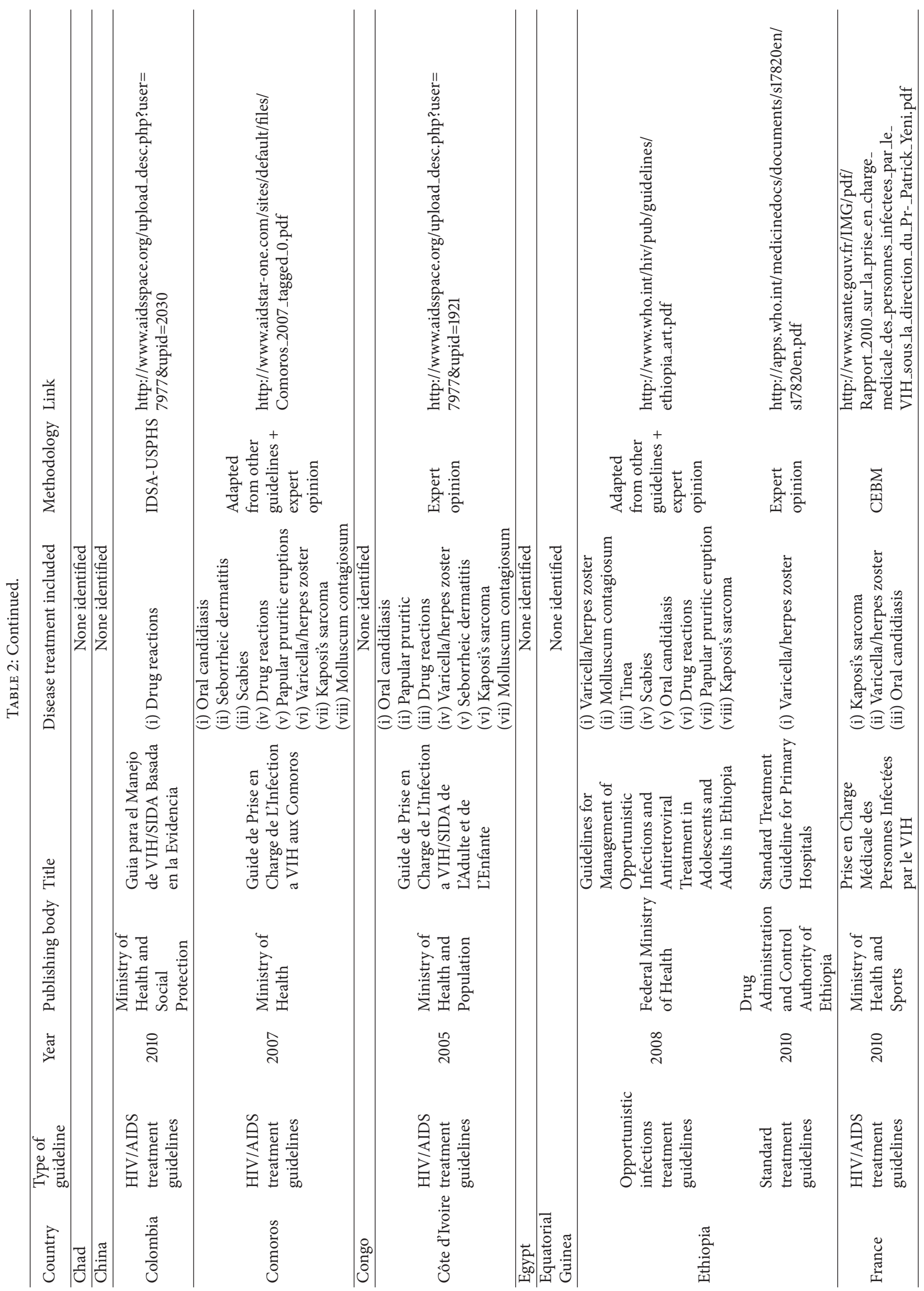




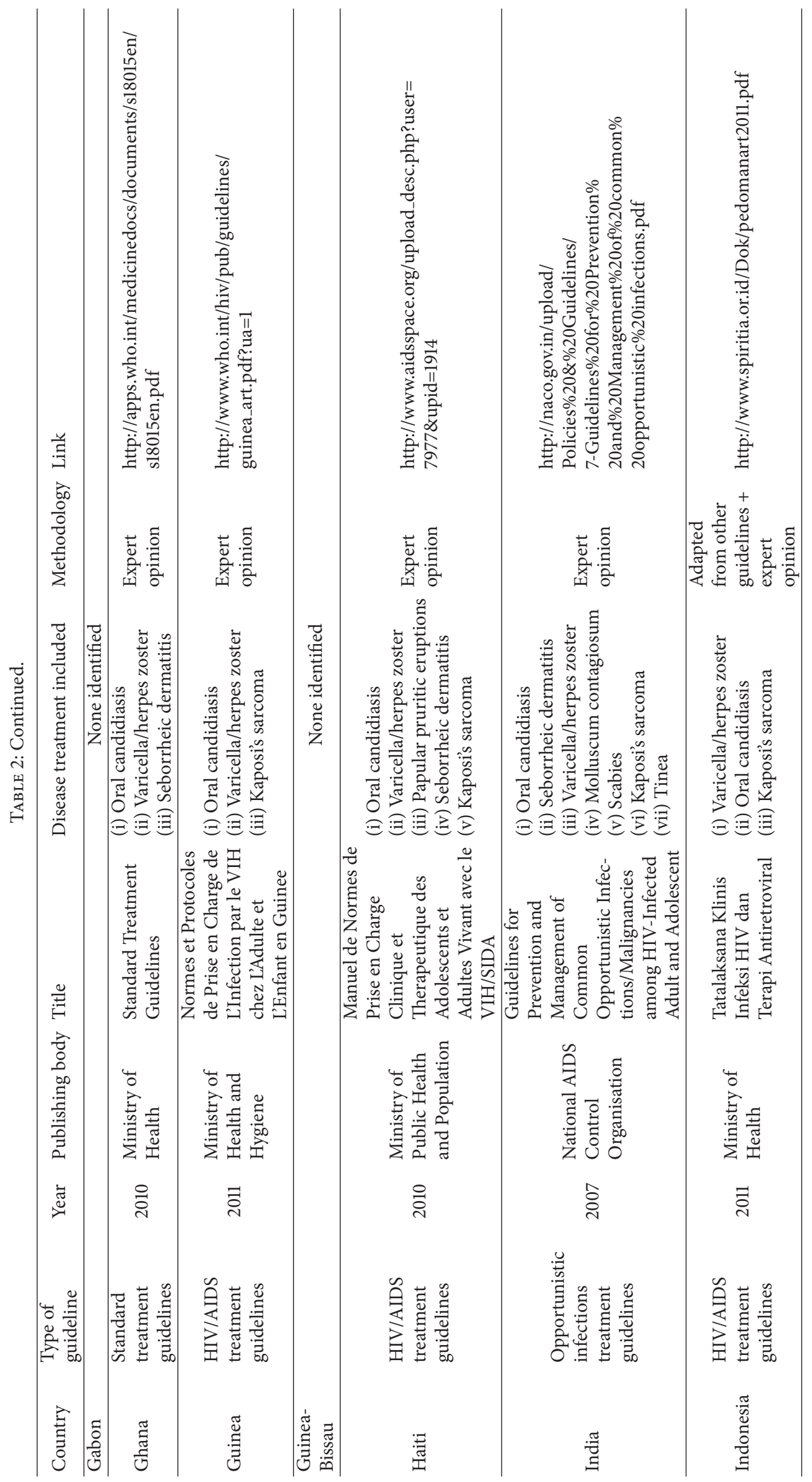




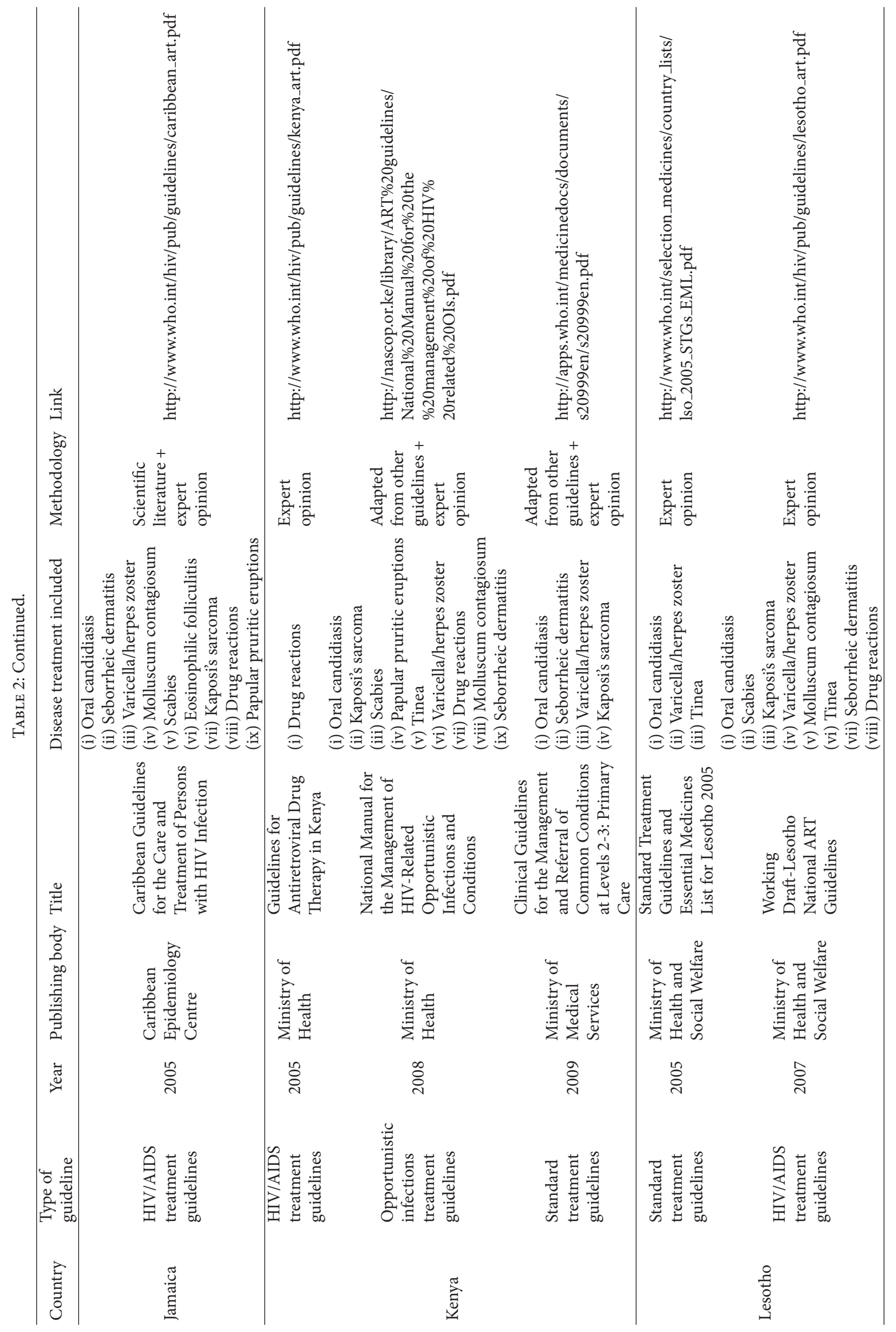




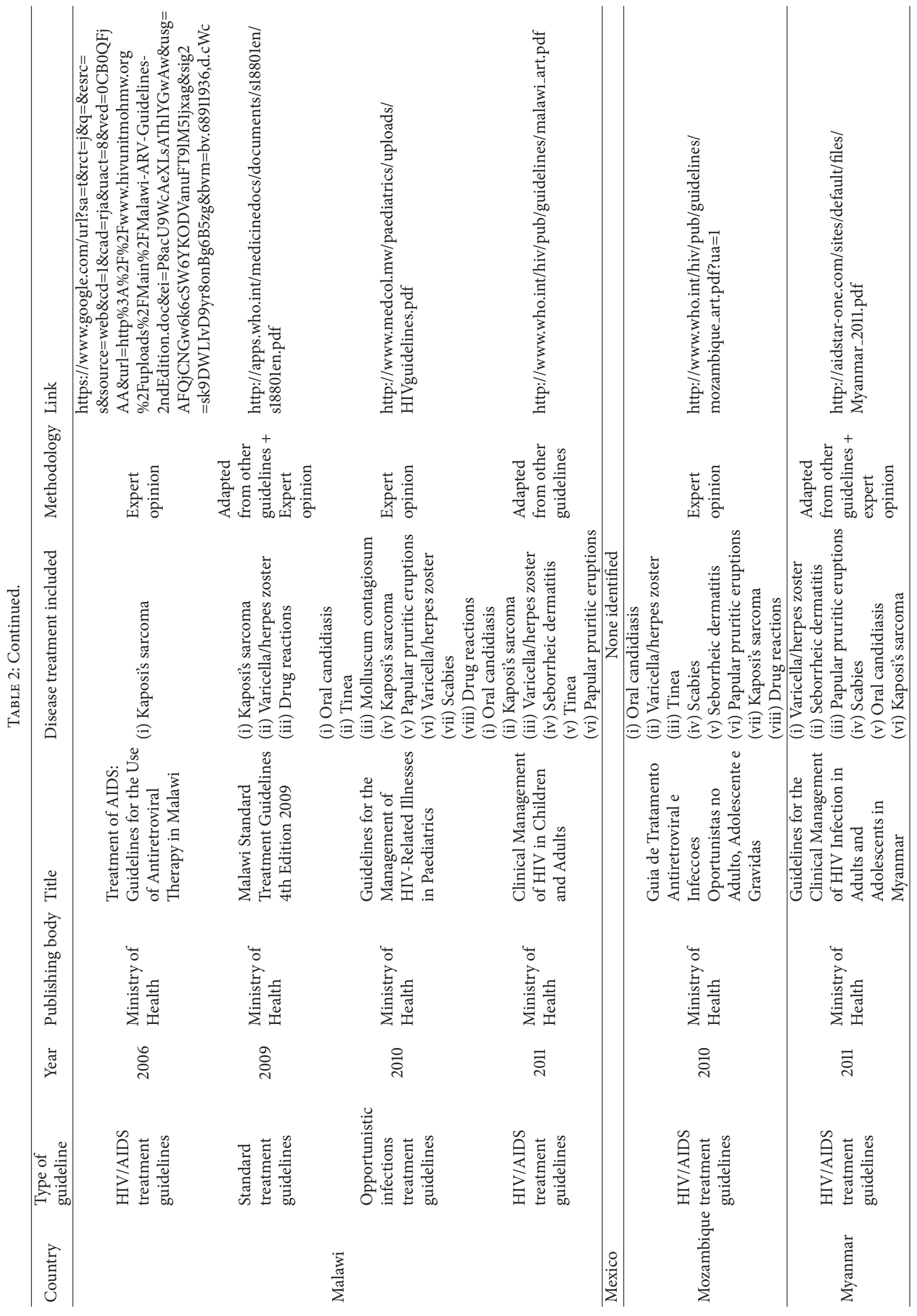




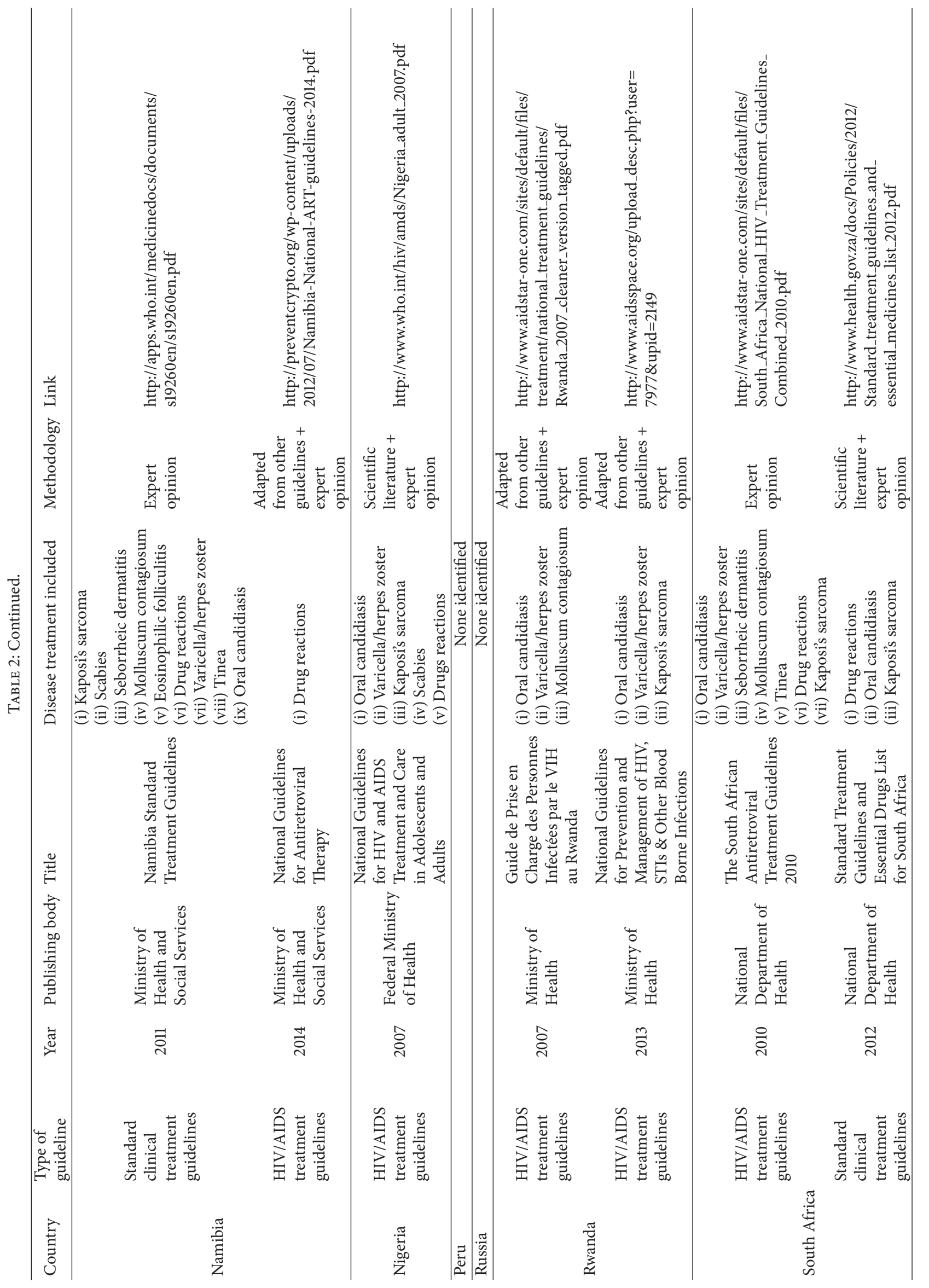




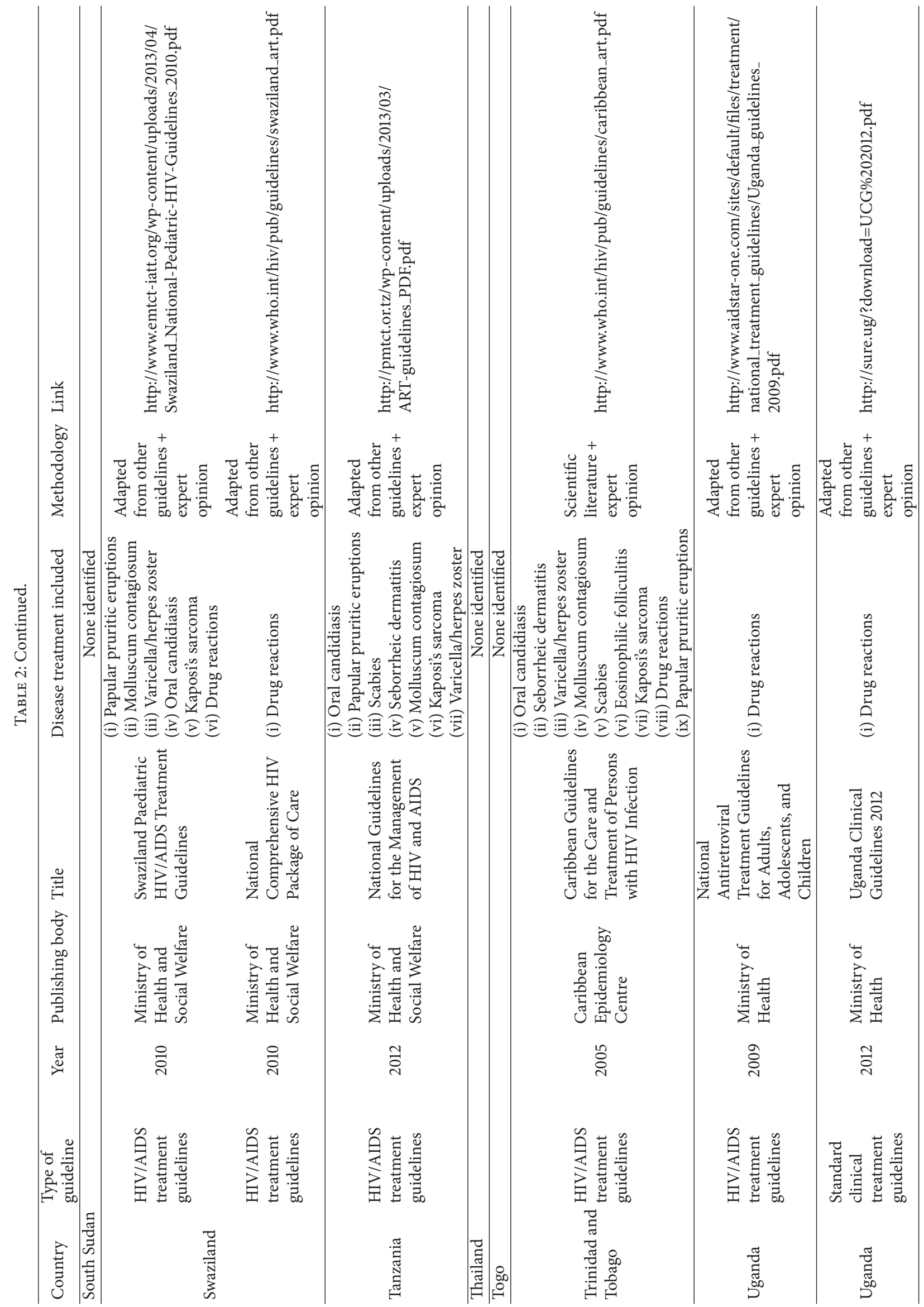




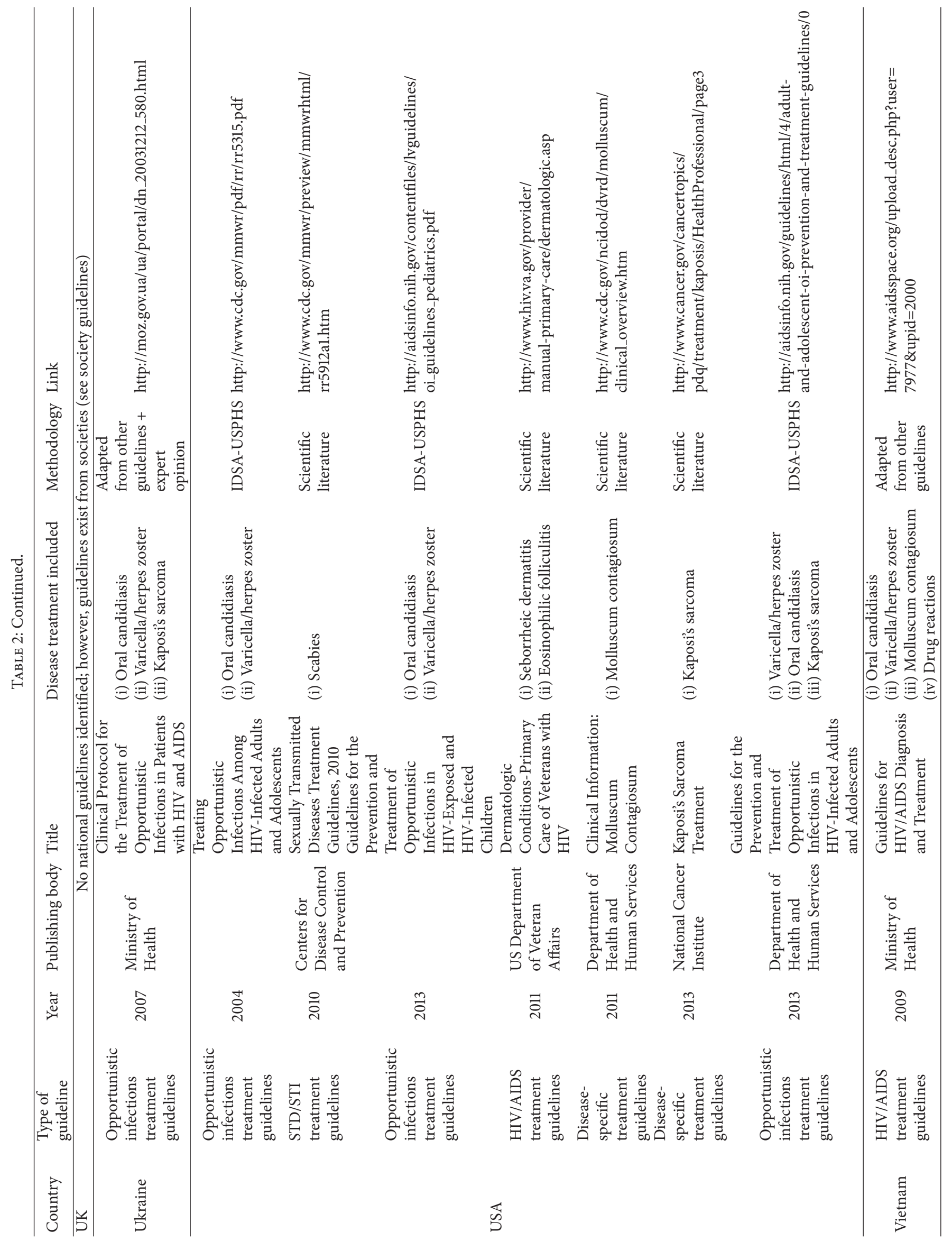




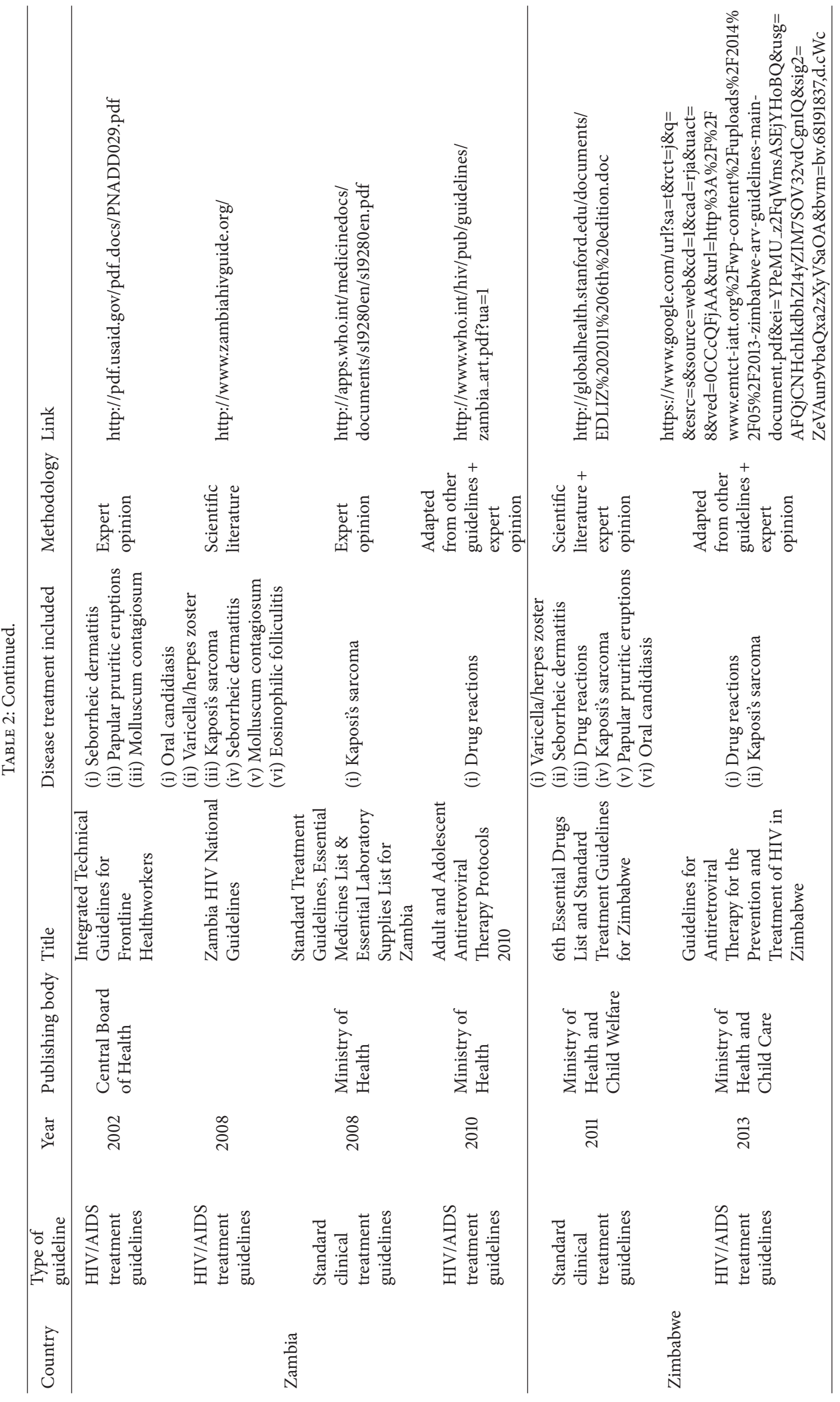




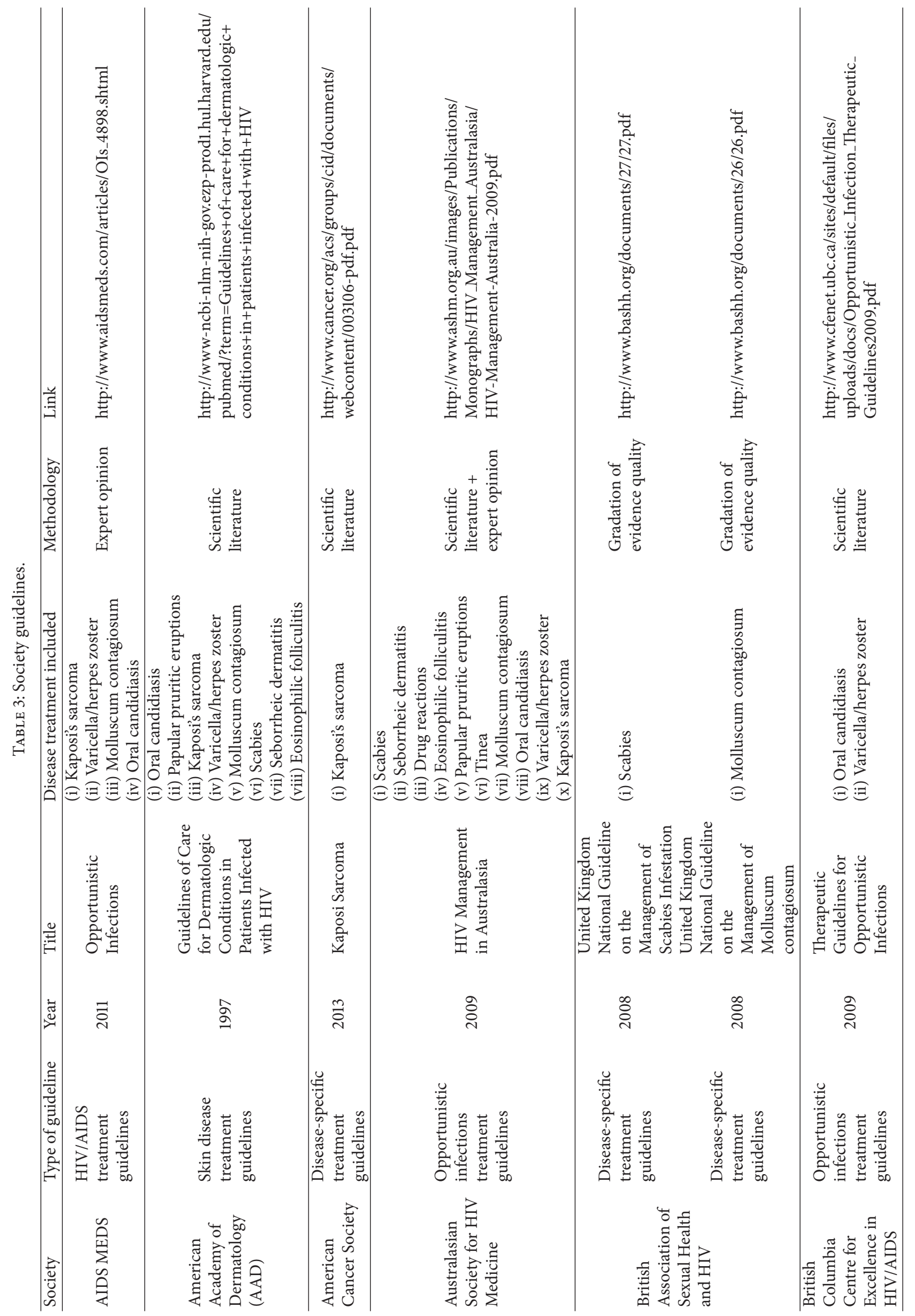




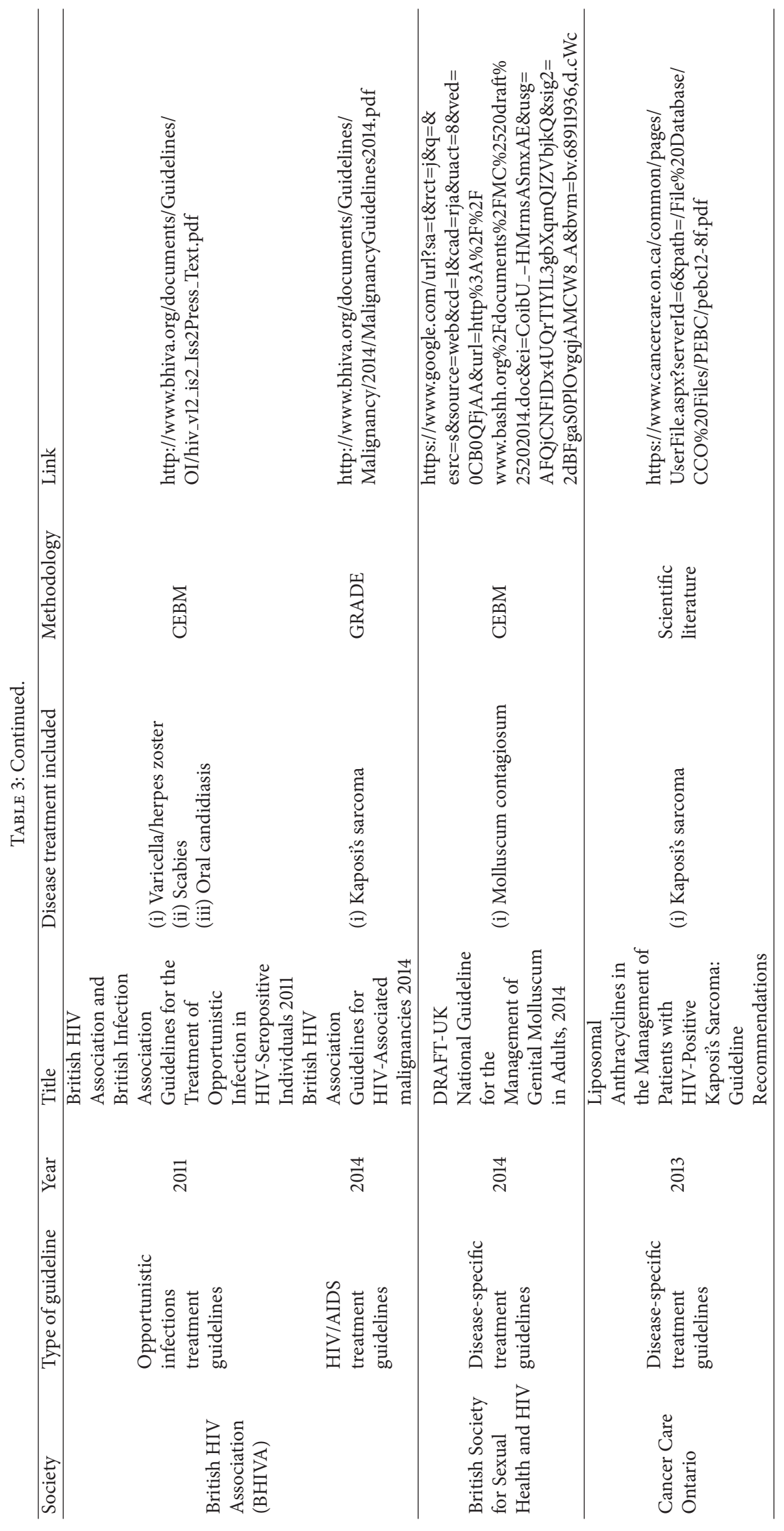




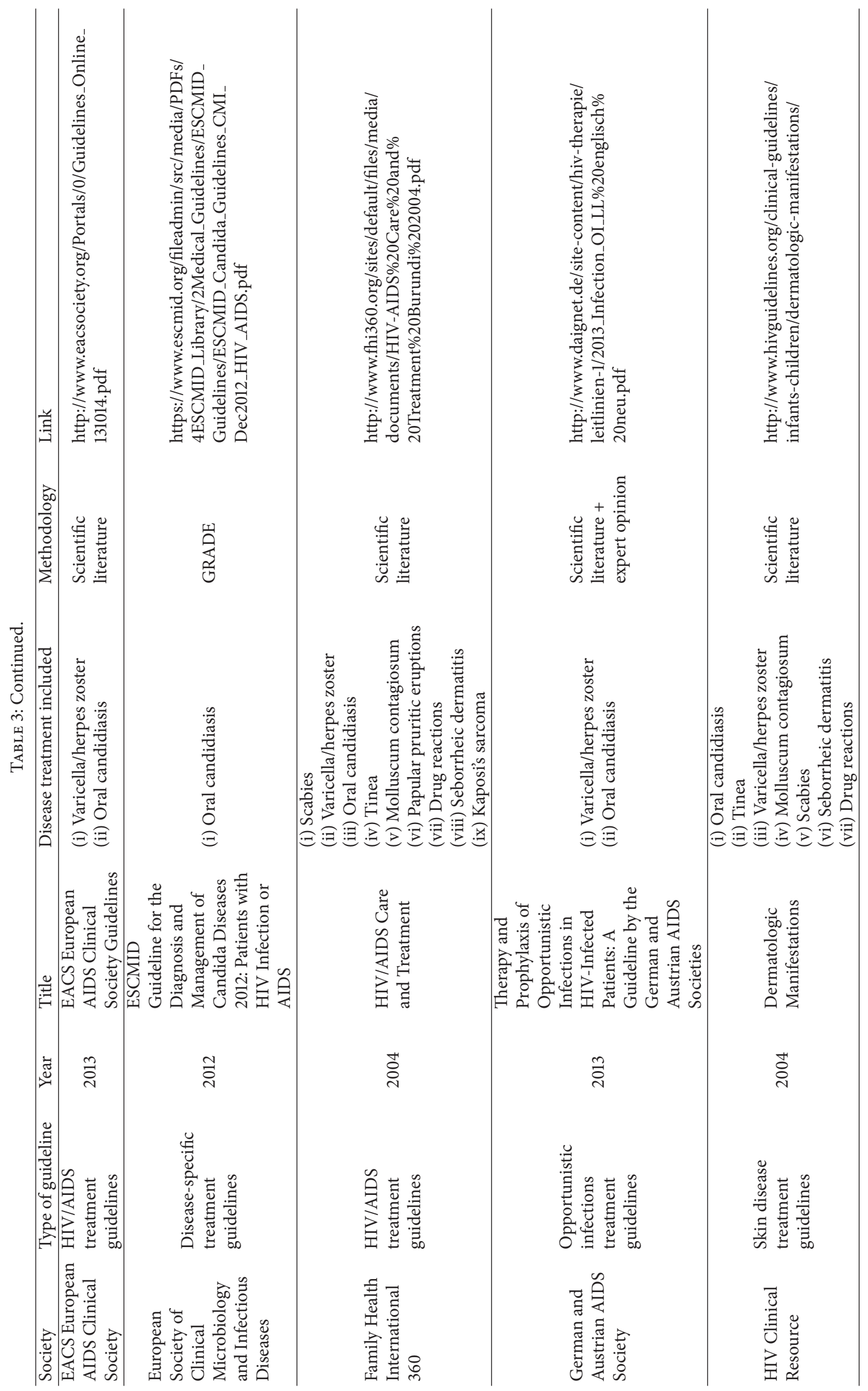




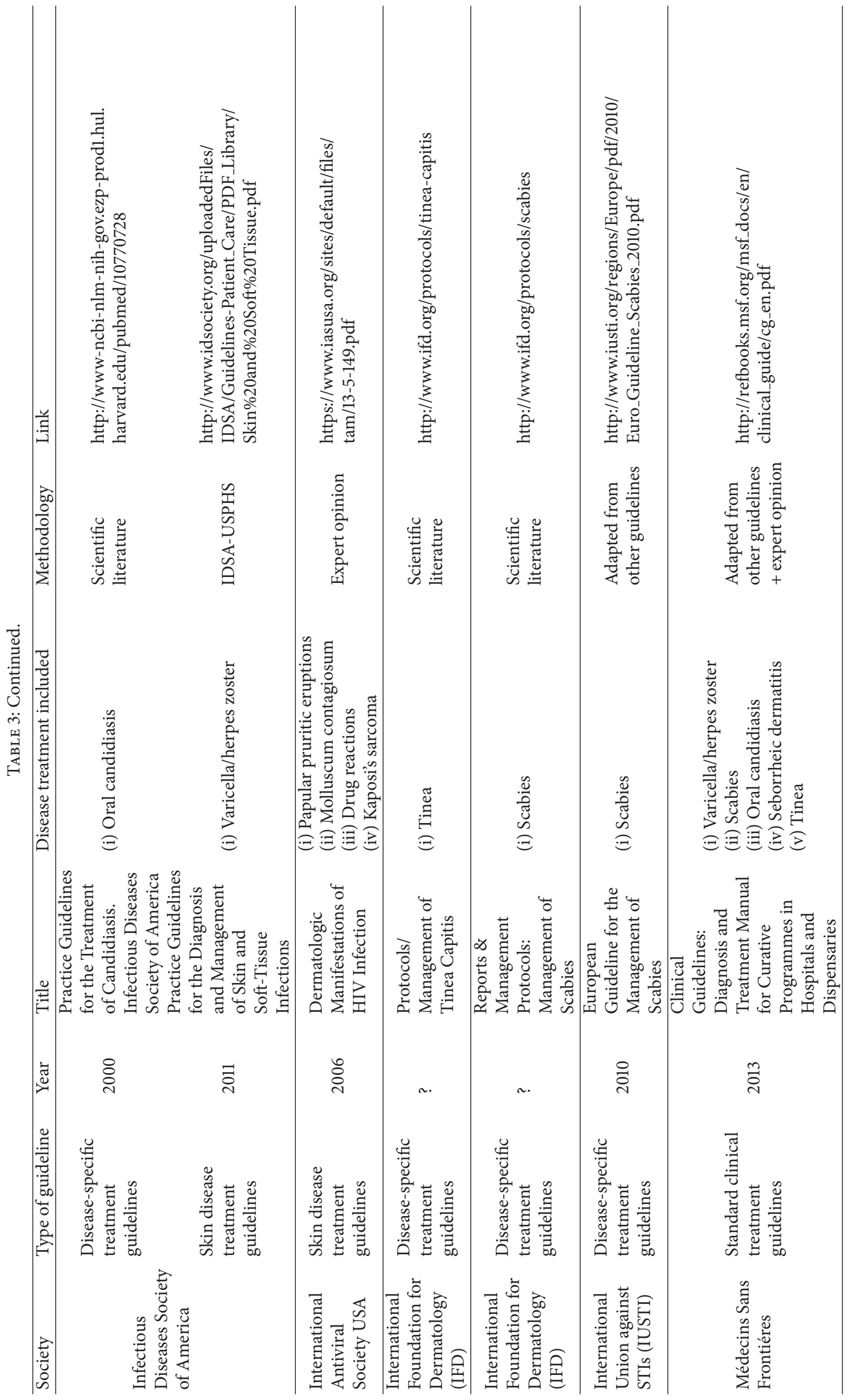




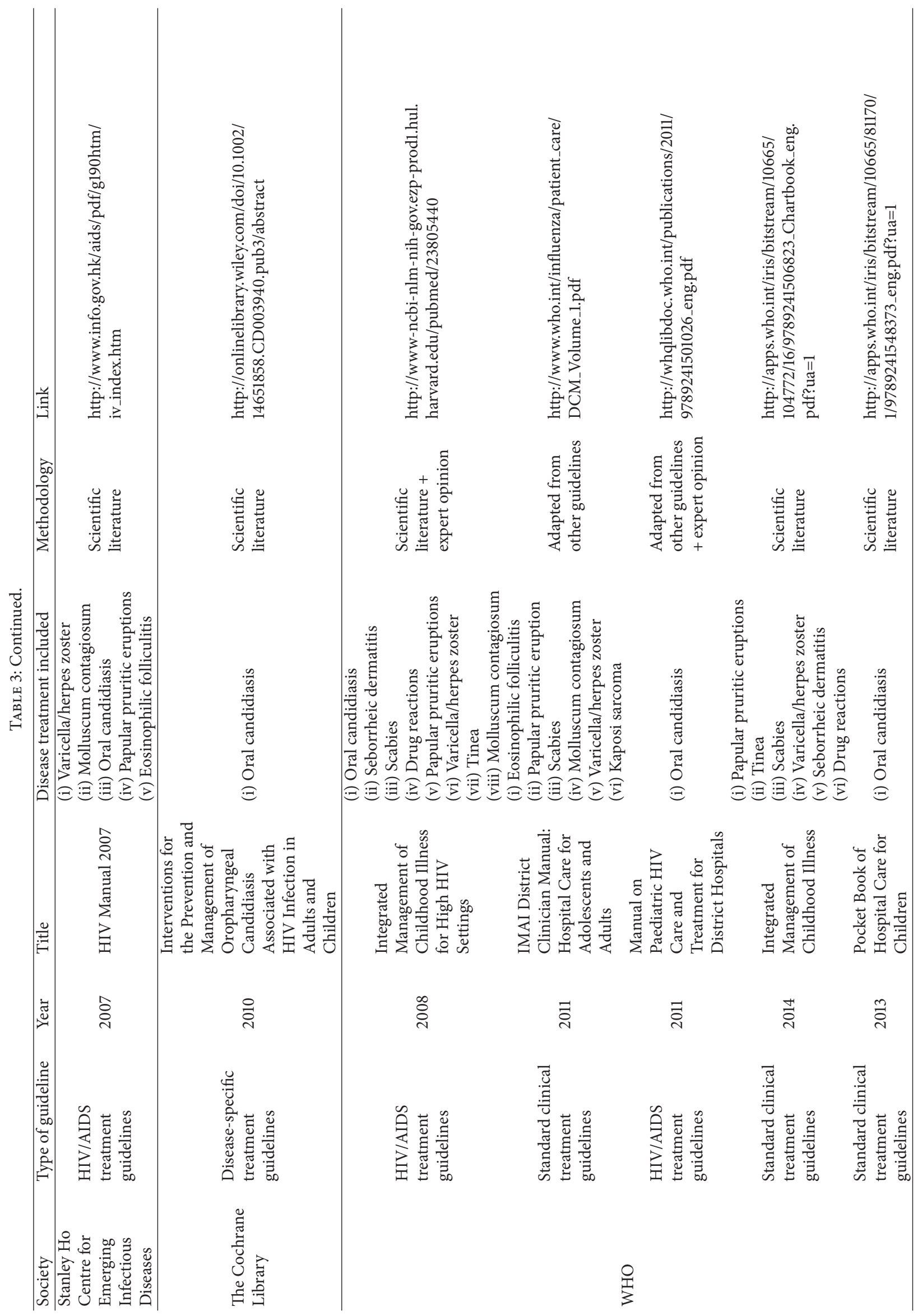


IDSA: Infectious Disease Society of America

STDs: Sexually transmitted infections

TB: Tuberculosis

USPHS: US Public Health Service

USPSTF: US Preventative Services Task Force

WHO: World Health Organization.

\section{Competing Interests}

The authors have no competing interests to declare.

\section{Acknowledgments}

The authors would like to thank Philippa J. Easterbrook and Amanda Costa for their valuable discussions and suggestions. Dr. Esther E. Freeman is supported by T32 AR007098.

\section{References}

[1] UNAIDS, The gap report, 2014, http://www.unaids.org/en/ media/unaids/contentassets/documents/unaidspublication/2014/ UNAIDS_Gap_report_en.pdf.

[2] R. J. Hay, N. E. Johns, H. C. Williams et al., “The global burden of skin disease in 2010: an analysis of the prevalence and impact of skin conditions," Journal of Investigative Dermatology, vol. 134, no. 6, pp. 1527-1534, 2014.

[3] E. Tschachler, P. R. Bergstresser, and G. Stingl, "HIV-related skin diseases," The Lancet, vol. 348, no. 9028, pp. 659-663, 1996.

[4] E. N. Nnoruka, J. C. Chukwuka, and B. Anisuiba, "Correlation of mucocutaneous manifestations of HIV/AIDS infection with CD4 counts and disease progression," International Journal of Dermatology, vol. 46, supplement 2, pp. 14-18, 2007.

[5] V. Mendiratta, S. Mittal, A. Jain, and R. Chander, "Mucocutaneous manifestations in children with human immunodeficiency virus infection," Indian Journal of Dermatology, Venereology and Leprology, vol. 76, no. 5, pp. 458-466, 2010.

[6] S. A. Coopman, R. A. Johnson, R. Platt, and R. S. Stern, "Cutaneous disease and drug reactions in HIV infection," The New England Journal of Medicine, vol. 328, no. 23, pp. 1670-1674, 1993.

[7] J. Hu, K. McKoy, A. Papier et al., "Dermatology and HIV/AIDS in Africa," Journal of Global Infectious Diseases, vol. 3, no. 3, pp. 275-280, 2011.

[8] G. Schmid-Ott, B. Jaeger, H. W. Kuensebeck, R. Ott, and F. Lamprecht, "Dimensions of stigmatization in patients with psoriasis in a 'Questionnaire on Experience with Skin Complaints,' Dermatology, vol. 193, no. 4, pp. 304-310, 1996.

[9] T. A. Maurer, "Dermatologic manifestations of HIV infection," Topics in HIV Medicine, vol. 13, no. 5, pp. 149-154, 2005.

[10] M. E. Garman and S. K. Tyring, "The cutaneous manifestations of HIV infection," Dermatologic Clinics, vol. 20, no. 2, pp. 193208, 2002.

[11] S. Lowe, R. A. Ferrand, R. Morris-Jones et al., "Skin disease among human immunodeficiency virus-infected adolescents in Zimbabwe: a strong indicator of underlying HIV infection," Pediatric Infectious Disease Journal, vol. 29, no. 4, pp. 346-351, 2010.

[12] R. Hay, S. E. Bendeck, S. Chen et al., "Skin diseases," in Disease Control Priorities in Developing Countries, D. T. Jamison, J. G. Breman, A. R. Measham et al., Eds., chapter 37, The
International Bank for Reconstruction and Development/The World Bank Group, Washington, DC, USA, 2006.

[13] M. Waugh, "Skin diseases-clinical indicator of immune status in HIV infection," International Journal of Dermatology, vol. 44, no. 8, pp. 705-706, 2005.

[14] A. M. Cook, I. G. Finlay, A. G. K. Edwards et al., "Efficiency of searching the grey literature in palliative care," Journal of Pain and Symptom Management, vol. 22, no. 3, pp. 797-801, 2001.

[15] UNAIDS, HIV Estimates with Uncertainty Bounds. UNAIDS Report on the Global AIDS Epidemic-2013, 2014, http://www .unaids.org/en/dataanalysis/knowyourepidemic/epidemiologypublications/.

[16] WHO, Database of National HIV and TB Guidelines, 2005-2011, 2014, http://www.who.int/hiv/pub/national_guidelines/en/.

[17] USAID, AIDSTAR-One, National Treatment Guidelines, June 2014, https://aidsfree.usaid.gov/resources/guidance-data/ treatment.

[18] The Interagency Task Team on the Prevention and Treatment of HIV Infection in Pregnant Women, Mothers and Children, June 2014, http://www.emtct-iatt.org/.

[19] AIDSspace, "Library," 2014, http://www.aidsspace.org/library .php.

[20] Cochrane Review Group on HIV/AIDS, June 2014, http://hiv .cochrane.org/about-cochrane-hivaids-group.

[21] G. H. Guyatt, A. D. Oxman, G. E. Vist et al., "GRADE: an emerging consensus on rating quality of evidence and strength of recommendations," The British Medical Journal, vol. 336, no. 7650, pp. 924-926, 2008.

[22] The World Bank Group 2014, July 2014, http://data.worldbank .org/country.

[23] M. J. Rico, S. A. Myers, and M. R. Sanchez, "Guidelines of care for dermatologic conditions in patients infected with HIV. Guidelines/Outcomes Committee. American Academy of Dermatology," Journal of the American Academy of Dermatology, vol. 37, no. 3, part 1, pp. 450-472, 1997.

[24] S. G. Pahwa, E. L. Handelsman, and H. T. Shin, Dermatologic Manifestations. HIV Clinical Resource, August 2014, http:// www.hivguidelines.org/wp-content/uploads/p-derm-7.14.08 .pdf.

[25] A. D. Oxman, J. N. Lavis, and A. Fretheim, "Use of evidence in WHO recommendations," The Lancet, vol. 369, no. 9576, pp. 1883-1889, 2007.

[26] P. G. Shekelle, E. Ortiz, S. Rhodes et al., "Validity of the agency for healthcare research and quality clinical practice guidelines: how quickly do guidelines become outdated?" Journal of the American Medical Association, vol. 286, no. 12, pp. 1461-1467, 2001.

[27] World Health Organization, Guidelines on the Treatment of Skin and Oral HIV-Associated Conditions in Children and Adults, World Health Organization, 2014, http://www.who.int/ maternal_child_adolescent/documents/skin-mucosal-and-hiv/ en/. 


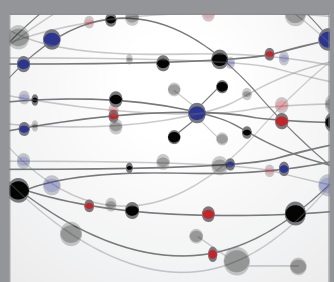

The Scientific World Journal
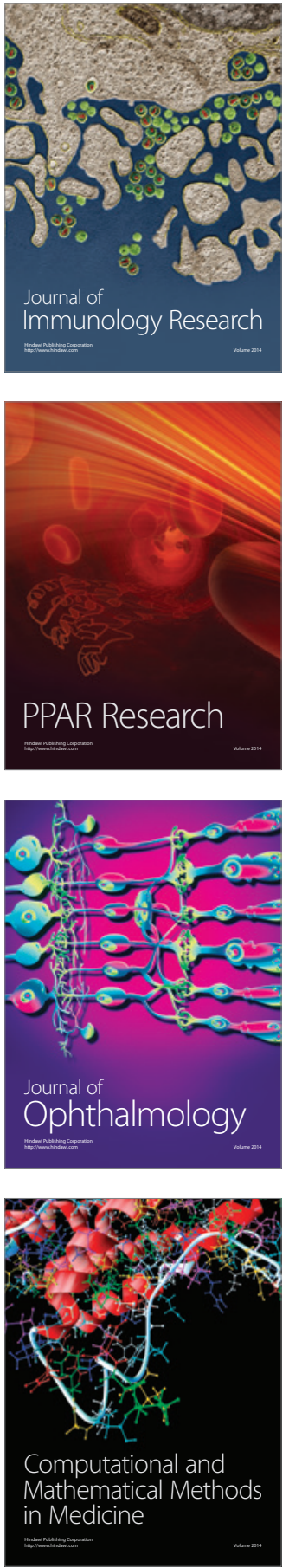

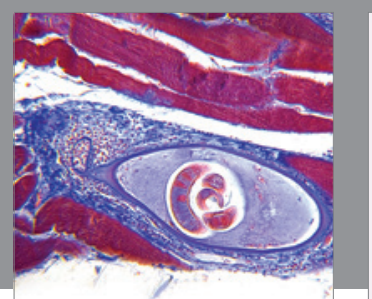

Gastroenterology Research and Practice

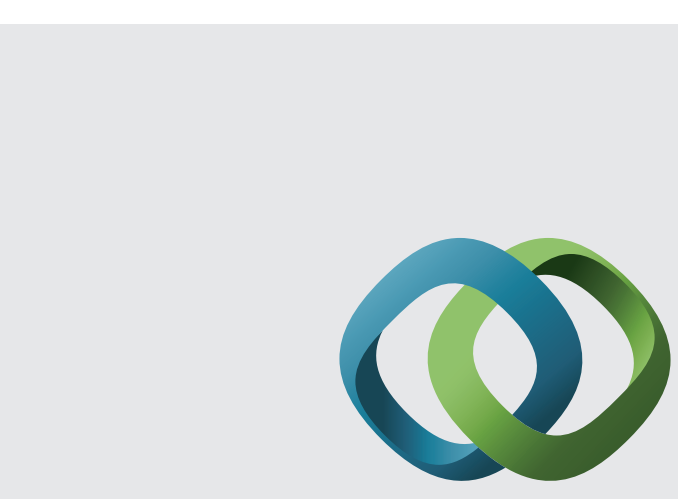

\section{Hindawi}

Submit your manuscripts at

http://www.hindawi.com
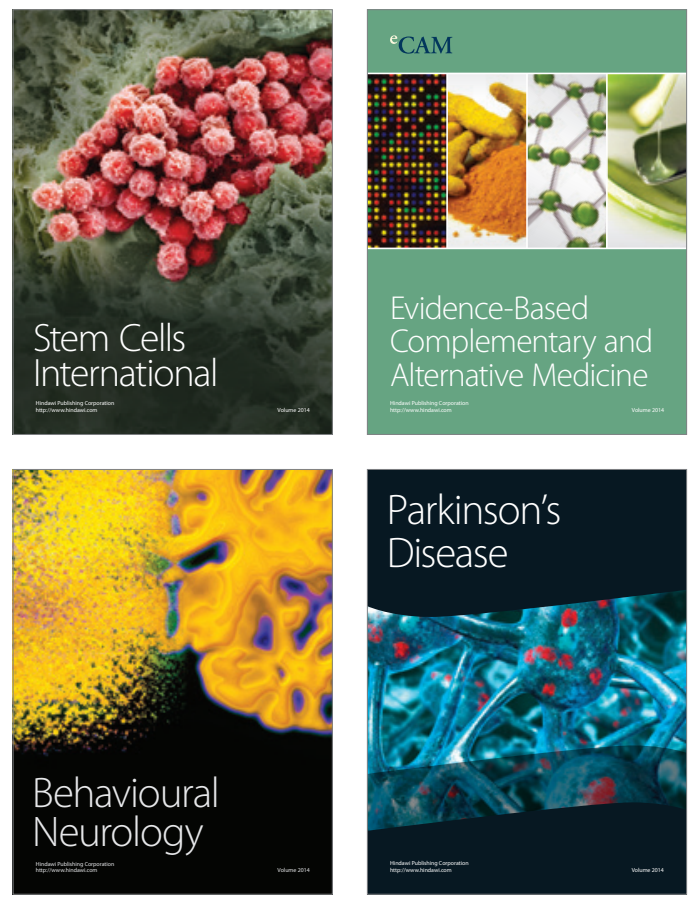
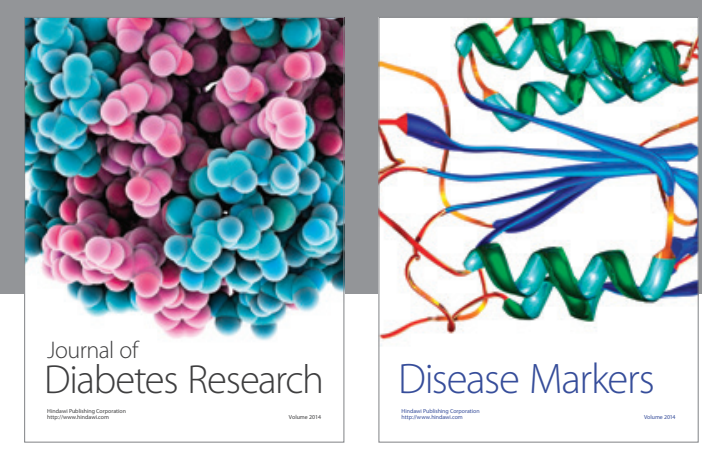

Disease Markers
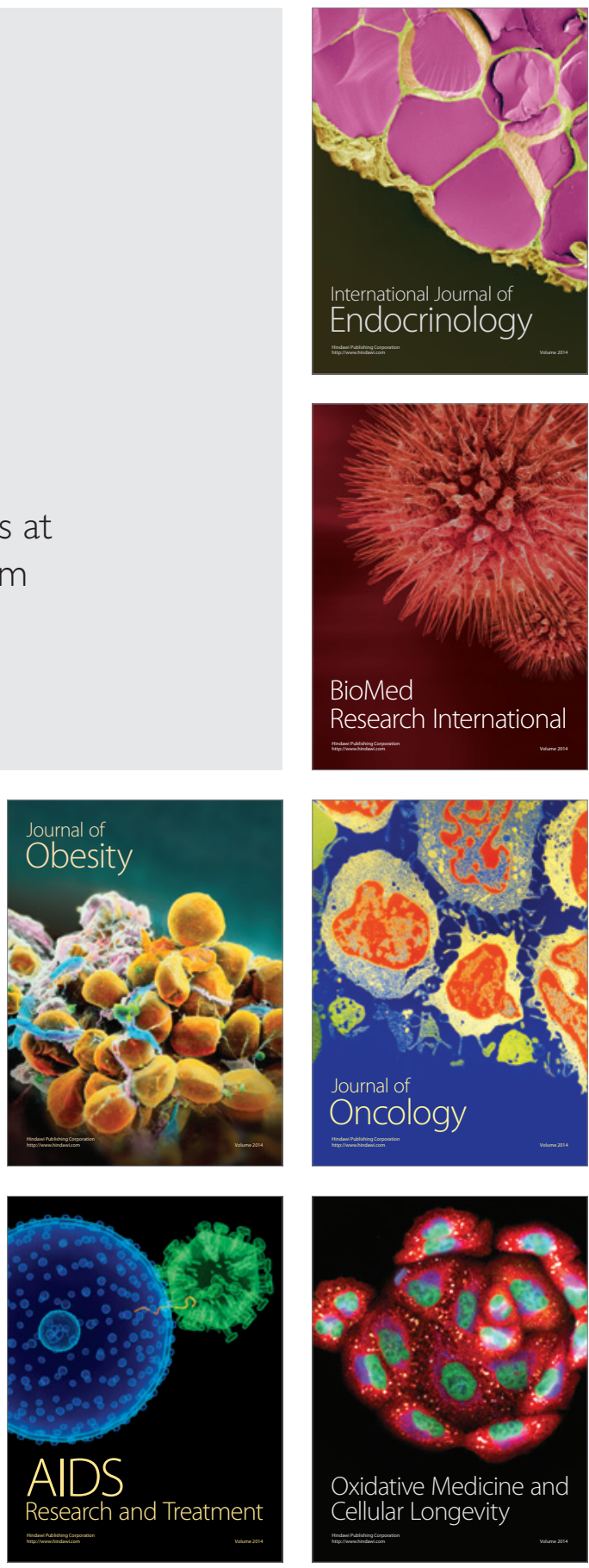